\title{
The fundamental group of the space of contact structures on the 3 -torus
}

\author{
HAnsjörg Geiges AND Mirko KlukAs
}

\begin{abstract}
We show that the fundamental group of the space of contact structures on the 3 -torus (based at the standard contact structure) is isomorphic to the integers.
\end{abstract}

\section{Introduction}

On the 3 -torus $T^{3}$ with coordinates $x, y, \theta \in \mathbb{R} / 2 \pi \mathbb{Z}$ the standard tight contact structure is given by

$$
\xi_{\mathrm{st}}=\operatorname{ker}(\cos \theta d x-\sin \theta d y)
$$

In [5] it was shown that the space $\Xi\left(T^{3}\right)$ of contact structures on $T^{3}$ has nontrivial topology: its fundamental group based at $\xi_{\text {st }}$ contains a subgroup isomorphic to the integers $\mathbb{Z}$. An alternative proof of that result was given by Bourgeois [1]. He also found nontrivial subgroups in higher homotopy groups of certain higher-dimensional contact manifolds. These subgroups are detected by their nontrivial action on the contact homology of the manifold in question.

Here we give a quick proof that $\mathbb{Z}$ constitutes the full fundamental group.

Theorem 1. The fundamental group of $\Xi\left(T^{3}\right)$, based at $\xi_{\mathrm{st}}$, is isomorphic to $\mathbb{Z}$.

The same statement holds with $\xi_{\text {st }}$ replaced by

$$
\xi_{n}=\operatorname{ker}(\cos (n \theta) d x-\sin (n \theta) d y)
$$

for any $n \in \mathbb{N}$.

Our proof of this theorem uses a homotopy exact sequence that will be described in Section 2. A similar approach was taken in [2] to show that the

2010 Mathematics Subject Classification. 53D35, 57R17. 
fundamental group of the space of contact structures on $S^{1} \times S^{2}$, based at the standard contact structure $\operatorname{ker}(z d \theta+x d y-y d x)$, is isomorphic to $\mathbb{Z}$.

Eliashberg [3] has shown that the space of tight contact structures on the 3-sphere fixed at a point is contractible; this will in fact be a key ingredient in the proof of Theorem 1. Beyond these results, very little is known about comparable homotopical questions.

In 2005, Bourgeois announced a proof of Theorem 1 that employs quite different methods: instead of relating the homotopy type of $\Xi\left(T^{3}\right)$ to that of the diffeomorphism resp. contactomorphism group of $\left(T^{3}, \xi_{\mathrm{st}}\right)$ via a homotopy exact sequence, a direct approach based on a cut-and-paste procedure along surfaces is used to show that $\pi_{1}\left(\Xi\left(T^{3}\right), \xi_{\text {st }}\right)$ is contained in $\mathbb{Z}$. We learned only recently that some details of this proof have been worked out by Fabien Ngô in his MSc thesis [14]. See also the comments following Proposition 2.

\section{A homotopy exact sequence}

Let $\left(M, \xi_{0}\right)$ be a closed contact manifold. Write $\Xi_{0}$ for the component of the space of contact structures on $M$ containing $\xi_{0}$. By Diff 0 we denote the identity component of the diffeomorphism group of $M$, and by $\mathrm{Cont}_{0}$ its subgroup of contactomorphisms of $\xi_{0}$. In other words, Cont $t_{0}$ is the group of those contactomorphisms that are isotopic via diffeomorphisms to the identity. In particular, Cont ${ }_{0}$ is not, in general, connected. As shown in [5], the inclusion map $i:$ Cont $_{0} \rightarrow$ Diff $_{0}$ and the map

$$
\begin{aligned}
\sigma: \operatorname{Diff}_{0} & \longrightarrow \Xi_{0} \\
\phi & \longmapsto \phi_{*} \xi_{0}
\end{aligned}
$$

induce a homotopy exact sequence

$$
\cdots \stackrel{\Delta}{\longrightarrow} \pi_{k}\left(\text { Cont }_{0}\right) \stackrel{i_{\#}}{\longrightarrow} \pi_{k}\left(\text { Diff }_{0}\right) \stackrel{\sigma_{\#}}{\longrightarrow} \pi_{k}\left(\Xi_{0}\right) \stackrel{\Delta}{\longrightarrow} \pi_{k-1}\left(\text { Cont }_{0}\right) \stackrel{i_{\#}}{\longrightarrow} \cdots
$$

On $M=T^{3}$ we have the loops $\gamma_{1}, \gamma_{2}, \gamma_{3}$ of diffeomorphisms, defined by

$$
\begin{array}{ll}
\gamma_{1}(s)(x, y, \theta)=(x+2 \pi s, y, \theta), & s \in[0,1] \\
\gamma_{2}(s)(x, y, \theta)=(x, y+2 \pi s, \theta), & s \in[0,1], \\
\gamma_{3}(s)(x, y, \theta)=(x, y, \theta+2 \pi s), & s \in[0,1] .
\end{array}
$$

The homotopy classes $\left[\gamma_{1}\right],\left[\gamma_{2}\right],\left[\gamma_{3}\right]$ of these three loops generate $\pi_{1}\left(\right.$ Diff $\left._{0}\right) \cong$ $\mathbb{Z}^{3}$; cf. [10]. The diffeomorphisms $\gamma_{1}(s), \gamma_{2}(s)$ of $T^{3}$ are contactomorphisms 
of $\xi_{\text {st }}$, so the subgroup $\mathbb{Z}^{2}+\{0\} \subset \mathbb{Z}^{3}$ generated by $\left[\gamma_{1}\right]$ and $\left[\gamma_{2}\right]$ lies in the image of

$$
i_{\#}: \pi_{1}\left(\text { Cont }_{0}\right) \longrightarrow \pi_{1}\left(\operatorname{Diff}_{0}\right),
$$

and hence in the kernel of

$$
\sigma_{\#}: \pi_{1}\left(\operatorname{Diff}_{0}\right) \longrightarrow \pi_{1}\left(\Xi_{0}\right)
$$

So the relevant part of the homotopy exact sequence for $\left(T^{3}, \xi_{0}\right)$ reduces to

$$
\mathbb{Z} \stackrel{\sigma_{\#}}{\longrightarrow} \pi_{1}\left(\Xi_{0}\right) \stackrel{\Delta}{\longrightarrow} \pi_{0}\left(\text { Cont }_{0}\right)
$$

where $\mathbb{Z}=\{(0,0)\}+\mathbb{Z} \subset \mathbb{Z}^{3}$ is generated by $\left[\gamma_{3}\right]$. As shown in $[5], \sigma_{\#}(\mathbb{Z})$ is an infinite cyclic subgroup of $\pi_{1}\left(\Xi_{0}\right)$.

\section{Proof of Theorem 1}

Theorem 1 is now an immediate consequence of the following result.

Proposition 2. For $\left(T^{3}, \xi_{\mathrm{st}}\right)$, the space Cont $_{0}$ is connected, i.e., every contactomorphism of $\left(T^{3}, \xi_{\mathrm{st}}\right)$ that is topologically isotopic to the identity is also isotopic to the identity via contactomorphisms.

This proposition, in turn, is a direct corollary of a result of Giroux [9, Théorème 4]. A proof of Theorem 1 based on this line of reasoning was given in [6]. Unfortunately, the published proof of Giroux's result is incomplete. (The error occurs in [9, Proposition 10]. A counterexample to that proposition has been constructed by Patrick Massot in his thesis [13]. The proofs of Giroux's main results, though, can be fixed using the methods of that thesis. The relevant results of Massot extend ideas of Ghiggini.) Here we present a proof of Proposition 2 based on a result of Ghiggini [7]. For the language of convex surfaces in the sense of Giroux see [8] or [4].

Proof of Proposition 2. The 2-torus $T:=\{y=0\}$ in $T^{3}$ is a vertical torus in standard form in the sense of [7]: a convex torus (transverse to the contact vector field $\partial_{y}$ ) with two dividing curves given by $\theta \in\{0, \pi\}$ and linear Legendrian ruling given by $\partial_{\theta}$ (the requirement "vertical" means that the Legendrian ruling has a nontrivial $\partial_{\theta}$-component). Likewise, the 2 -torus $S:=\{x=0\}$ is such a vertical torus.

Now let $\phi \in$ Cont $_{0}$ be given. Then $\phi(T)$ is smoothly isotopic to $T$, and by a result of Ghiggini [7, Lemma 6.5] it is contact isotopic to $T$. 
Ghiggini's work is based on isotopy discretization [12, Lemma 3.10], an idea going back to Colin. So $\phi$ is isotopic in Cont $_{0}$ to a contactomorphism $\phi^{\prime}$ fixing $T$. By the isotopy extension theorem for surfaces in contact 3manifolds, cf. [4, Theorem 2.6.13], we may in fact assume that $\phi^{\prime}$ fixes $T$ pointwise.

The second vertical torus $S$ intersects $T$ in a Legendrian ruling curve, and it is smoothly isotopic to the torus $\phi^{\prime}(S)$ sharing this property. By [7, Lemma 6.3], $\phi^{\prime}(S)$ is contact isotopic to $S$ (keeping $T$ fixed). Thus, we can isotope $\phi^{\prime}$ in Cont $_{0}$ to a contactomorphism $\phi^{\prime \prime}$ fixing both $T$ and $S$ pointwise.

The solid torus $T^{3} \backslash(S \cup T)$ becomes, after edge-rounding [11, Lemma 3.11], a standard tight solid torus with two dividing curves of slope -1 . Now consider a convex meridional disc $D$ in this solid torus and its image under $\phi^{\prime \prime}$. Note that the dividing set of $D$ consists of a single properly embedded arc. By isotopy discretization one sees that $\phi^{\prime \prime}(D)$ is contact isotopic to $D$, so we can now isotope $\phi^{\prime \prime}$ in Cont $_{0}$ to $\phi^{\prime \prime \prime}$ fixing $S, T$ and $D$.

The complement $T^{3} \backslash(S \cup T \cup D)$, after edge-rounding, is a standard tight 3-ball $\left(B^{3}, \xi_{\mathrm{st}}\right)$, and $\phi^{\prime \prime \prime}$ may be regarded as a contactomorphism of $\left(B^{3}, \xi_{\mathrm{st}}\right)$ fixing the boundary. According to Eliashberg [3, Theorem 2.4.2], the space of tight contact structures on $B^{3}$ with fixed boundary condition is contractible, in particular, its fundamental group is trivial. A homotopy exact sequence as in the previous section then shows that the space of contactomorphisms of $\left(B^{3}, \xi_{\text {st }}\right)$ fixed at the boundary (and topologically isotopic to the identity) is connected. This concludes the proof of the proposition.

Remark. In the last part of the foregoing proof, we only had to deal with contactomorphisms of $B^{3}$ (fixed at the boundary) that are known by assumption to be topologically isotopic to the identity. In fact, however, by Hatcher's proof of the Smale conjecture (saying that the diffeomorphism group of $S^{3}$ retracts to the orthogonal group of isometries) this topological condition is always satisfied: the full diffeomorphism group of $B^{3}$ relative to the boundary is contractible. It follows that the same is true for the contactomorphism group of $\left(B^{3}, \xi_{\mathrm{st}}\right)$ relative to the boundary, cf. [9, Théorème 17].

\section{Acknowledgments}

The authors are partially supported by DFG grant GE 1245/2-1. 


\section{References}

[1] F. Bourgeois, Contact homology and homotopy groups of the space of contact structures, Math. Res. Lett. 13 (2006), 71-85.

[2] F. Ding and H. Geiges, The diffeotopy group of $S^{1} \times S^{2}$ via contact topology, Compos. Math. 146 (2010), 1096-1112.

[3] Y. Eliashberg, Contact 3-manifolds twenty years since J. Martinet's work, Ann. Inst. Fourier (Grenoble) 42 (1992), 165-192.

[4] H. Geiges, An introduction to contact topology, Cambridge Studies in Advanced Mathematics, 109, Cambridge University Press, 2008.

[5] H. Geiges and J. Gonzalo Pérez, On the topology of the space of contact structures on torus bundles, Bull. Lond. Math. Soc. 36 (2004), 640-646.

[6] H. Geiges and J. Gonzalo Pérez, Fifteen years of contact circles and contact spheres, Acta Math. Vietnam 38 (2013), 145-164.

[7] P. Ghiggini, Linear Legendrian curves in $T^{3}$, Math. Proc. Camb. Philos. Soc. 140 (2006), 451-473.

[8] E. Giroux, Convexité en topologie de contact, Comment. Math. Helv. 66 (1991), 637-677.

[9] E. Giroux, Sur les transformations de contact au-dessus des surfaces, in 'Essays on Geometry and Related Topics' (É. Ghys et al., eds), 2, 329-350, Monographies de l'Enseignement Mathématique 38, Genève, 2001.

[10] A.E. Hatcher, Linearization in 3-dimensional topology, in 'Proc. Int. Congress of Mathematicians' (Helsinki, 1978), 463-468, Acad. Sci. Fennica, Helsinki, 1980.

[11] K. Honda, On the classification of tight contact structures I, Geom. Topol. 4 (2000), 309-368.

[12] K. Honda, Gluing tight contact structures, Duke Math. J. 115 (2002), 435-478.

[13] P. Massot, Sur quelques propriétés riemanniens des structures de contact en dimension trois, Thèse de doctorat, ENS Lyon, 2008.

[14] F. Ngô, Le groupe fondamental de l'espace des structures de contact tendues sur $T^{3}$, MSc thesis, Université Libre de Bruxelles, 2005. 
Mathematisches Institut, Universität ZU KÖLN

WEYERTAL 86-90, 50931 KÖLN

Germany

E-mail address: geiges@math.uni-koeln.de

E-mail address: mklukas@math.uni-koeln.de

Received OCtober 26, 2012 